\title{
Correction to: Intermarriage in the Twenty-First Century: New Perspectives
}

\author{
Bruce A. Phillips
}

\section{Correction to:}

Chapter 2 in: A. Dashefsky, I. M. Sheskin (eds.), American Jewish Year Book 2017, American Jewish Year Book 117, https://doi.org/10.1007/978-3-319-70663-4_2

In this version, in table 2.40, under heading "Single ancestry", in 1st column, 5 th row, the value has been changed from 94 to 0 . 